Supplementary Information for

\title{
Trace Key Mechanistic Features of the Arsenite Sequestration Reaction with Nanoscale Zero-Valent Iron
}

Li Cheng Kao ${ }^{a, \ddagger}$, Yang Ha ${ }^{a, \ddagger}$, Wan-Jou Chang ${ }^{b}$, Xuefei Fenga, Yifan $\mathrm{Ye}^{c}$, Jeng-Lung Chen ${ }^{d}$, Chih-Wen Paod, Feipeng Yanga, Catherine Zhue, Wanli Yanga, Jinghua Guo a,f, ${ }^{a}$, and Sofia Ya Hsuan Liou ${ }^{\mathrm{b}, \mathrm{g}, *}$

a Advanced Light Source, Lawrence Berkeley National Laboratory, Berkeley, California 94720, USA

b Department of Geosciences, National Taiwan University, Taipei 10617, Taiwan c National Synchrotron Radiation Laboratory, University of Science and Technology of China, Hefei, Anhui 230026, P. R. China

d National Synchrotron Radiation Research Center, Hsinchu 30076, Taiwan

e Molecular and Cellular Biology: Biochemistry, University of California, Berkeley, CA 94720, USA

f Department of Chemistry and Biochemistry, University of California, Santa Cruz, CA 95064, USA

g Research Center for Future Earth, National Taiwan University, Taipei 10617, Taiwan *Email: jguo@lbl.gov or yhliou@ntu.edu.tw ¥L. C. K. and Y. H. contributed equally to this work. 


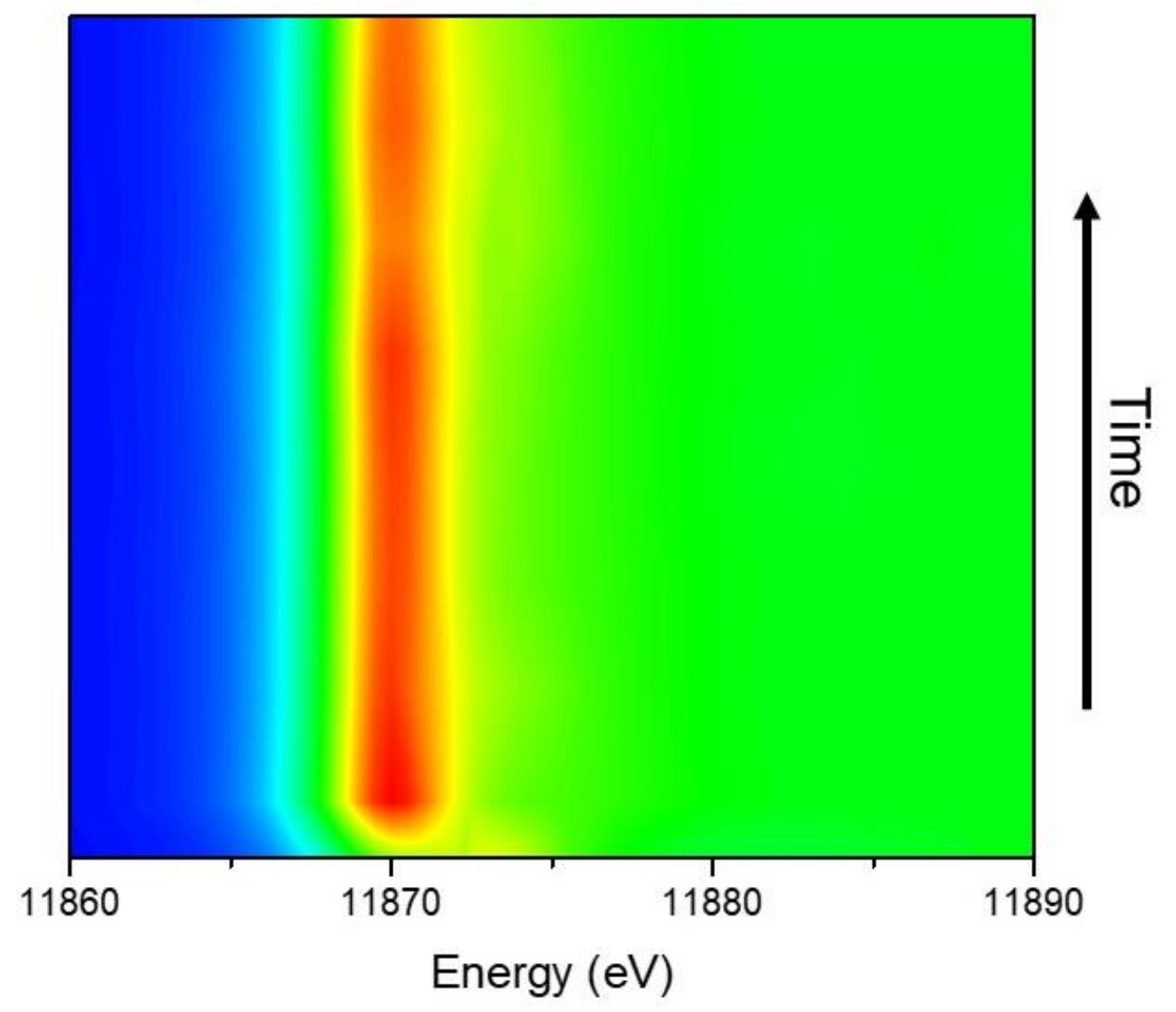

Figure S1. X-ray irradiation effect test 


\section{Note for in-situ experiment design.}

To design an in-situ experiment, it is essential to keep the system as simple as possible. The simple system could be also beneficial to do the DFT calculation. In this research, the lab-based batch experiments were implemented to simulate the synchrotron-based in-situ experiments. Therefore, it is important to set up the lab-based batch experiments as much like as the synchrotron-based in-situ experiments.

About the $\mathrm{pH}$ :

Second, because the synchrotron-based in-situ methodology is the main interpretative technique, especially in-situ XAS, it is crucial to choose the right indicators to discover the key mechanistic features of the As-nZVI sequestration reaction. In this work, the $\mathrm{As}^{\mathrm{III}}$ sequestration curve variation is the primary indicator to determine whether it worth to explore or not. The $\mathrm{pH}$ value of the lab-based $\mathrm{As}^{\mathrm{III}}$ sequestration experiments is listed below, but there is no obvious relationship between $\mathrm{pH}$ and $\mathrm{nZVI}$ concentration.

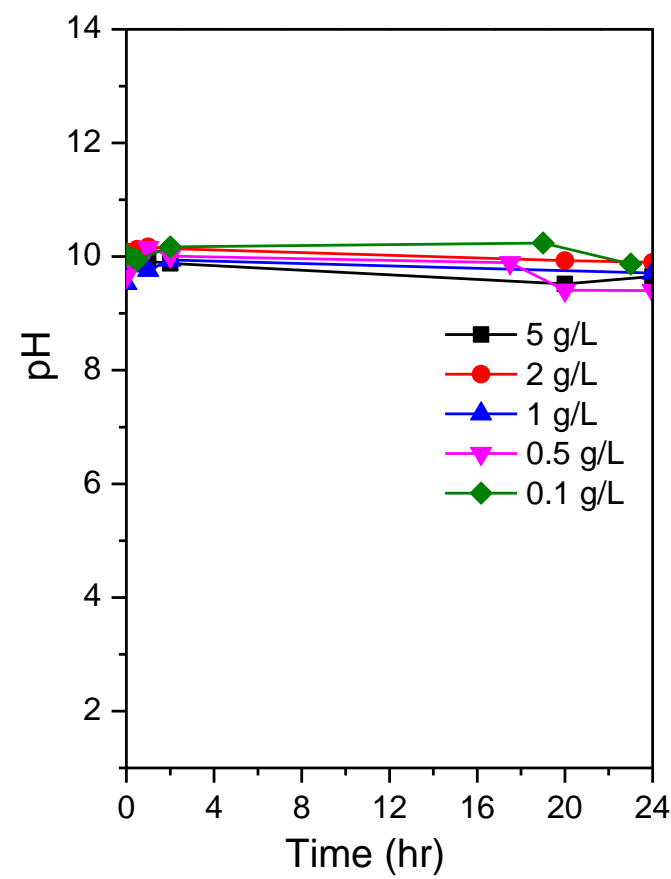

Figure S2. $\mathrm{pH}$ value of As ${ }^{\mathrm{III}}$ sequestration experiments at different $\mathrm{nZVI}$ concentration

About the oxidation-reduction potential:

According to Shi et al., there is considerable uncertainty about the meaning of oxidation-reduction potential measurements made in the presence of nZVI. All of the observed changes are the result of reactions between nZVI and the medium. The measured oxidation-reduction potential, which is a mixed potential composed of the 
weighted sum of Nernstian terms for each of the redox couples that are present at the electrode surface. Redox couples are likely to be in solutions containing various dissolved phase complexes and also involving in solid phase. It worth to design another in-situ cell to collect the solution properties (Eh, $\mathrm{pH}$, etc.) with XAS simultaneously, and use both data to characterize the reaction pathway. Although the Eh is not simultaneously recording with the XAS data, the As ${ }^{\mathrm{III}}$ sequestration curve variation can work as a similar indicator. Shi et al. found the oxidation-reduction potential is highly affected by the concentration of suspended nZVI. At high concentrations of nZVI, oxidation-reduction potential is dominated by direct interaction between the electrode and the nZVI particles, making the electrode relatively insensitive to changes in solution chemistry. Lower nZVI concentrations give a proportional response in oxidation-reduction potential, inducing from the mixture of contributions that includes adsorbed $\mathrm{nZVI}$ and the dissolved $\mathrm{H}_{2}$ and $\mathrm{Fe}^{\mathrm{II}}$ species that arise from corrosion of $\mathrm{nZVI}$. This result is coincidental with the As ${ }^{\mathrm{III}}$ sequestration curve variation and ICP analysis which listed below.
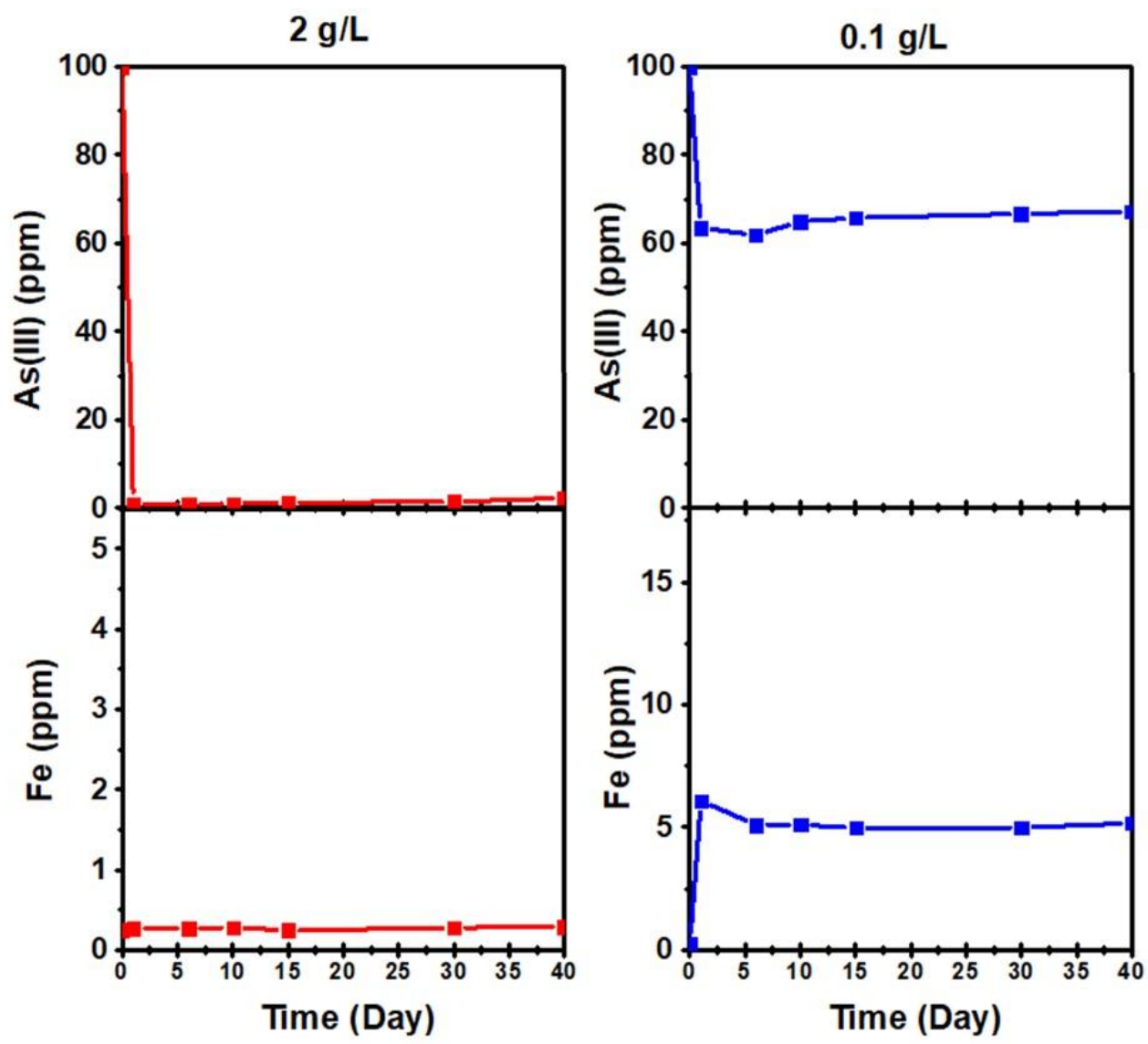

Figure S3. Dose effect on As"II sequestration curve and iron ICP

1. Zhenqing Shi, James T. Nurmi, Paul G. Tratnyek "Effects of Nano Zero-Valent Iron on Oxidation-Reduction Potential" Environ. Sci. Technol. 2011, 45, 1586-1592 
About purging gas:

As mentioned above, the synchrotron-based in-situ experiment has its own limitation and should be a possible set-up at the beamline endstation. Although there is no monitoring the real oxygen concentration of the in in-situ experimental solution, the $\mathrm{As}^{\mathrm{III}}$ sequestration curve variation can work as an indicator to predict the discrepancy. The As ${ }^{\mathrm{III}}$ sequestration experiment with different purging gas is listed below.
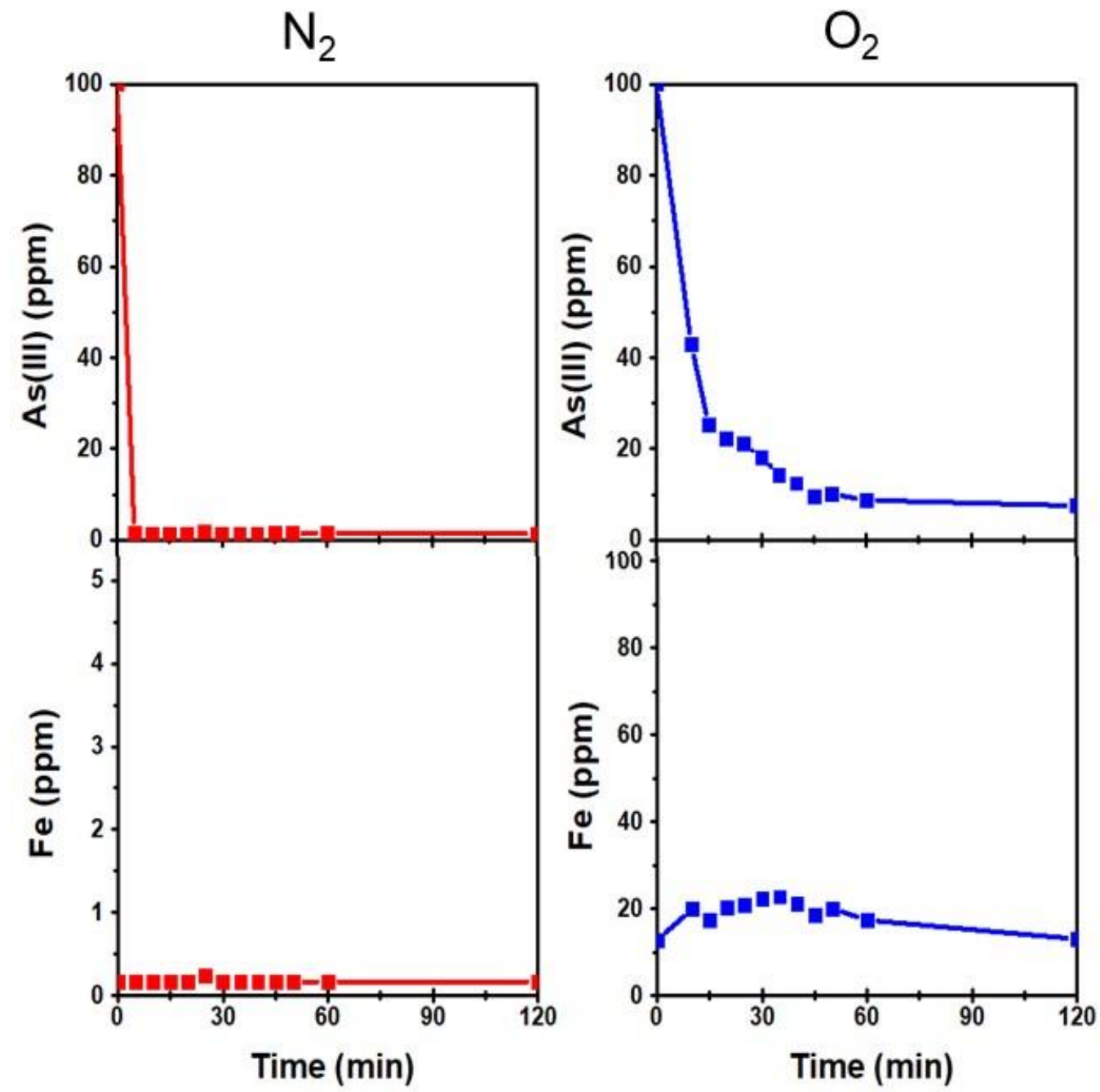

Figure S4. Purging gas effect on As'II sequestration curve and iron ICP 


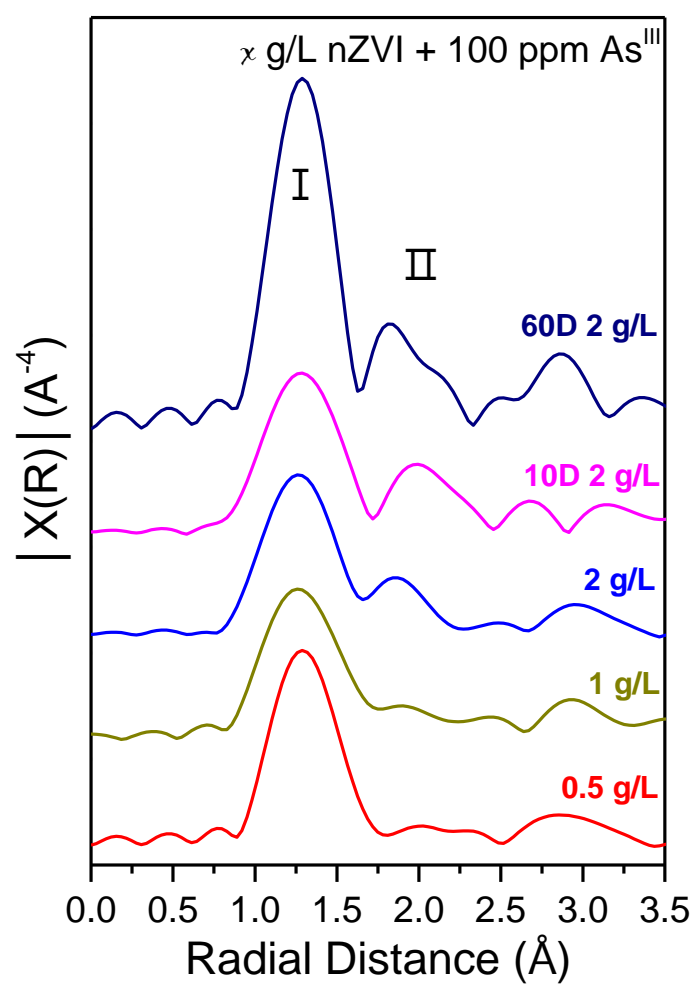

Figure S5. EXAFS of concentrated precipitate immediately collected from the real-time monitoring experiment

Note for EXAFS:

EXAFS raw data was done by aligning the data in absolute energy, correcting for the pre-edge slope, subtracting the background signal, normalizing by the edge step, converting to frequency $(\mathrm{k})$ space, and weighted by $k^{3}$.

EXAFS analysis which can provide the atomic environment of arsenic, as well as structural and chemical parameters, is an idea method to reveal long-term structural transformation. The Fourier transforms (FTs) of the As EXAFS of concentrated precipitate immediately collected from the real-time monitoring experiment are shown in Fig. S5. The amount of sample collected from $0.1 \mathrm{~g} / \mathrm{L}$ reaction is relative low and most likely not able to covert the low signal to noise data for FTs. The results of EXAFS analysis show that the steady-state products of $\mathrm{As}^{\mathrm{III}}$ formed through the various reaction times and nZVI doses have different structures. Area I as the first shell has contributions from bridging and terminal $\mathrm{O}$ atoms at $1.2 \AA$. This distance is in agreement with values reported in the literature for $\mathrm{As}{ }^{\mathrm{III}}$ species. Area II with distances around $1.8 \AA$ is more obvious in the long-term reaction system ${ }^{1}$. This second shell is reasonable to assign as the coordination shell of $\mathrm{As}^{\mathrm{V}}-\mathrm{O}$ bonding, which can be a two oxygen shell model at distances characteristic of the arsenite trigonal pyramidal and arsenate tetrahedral 
geometries. At distances greater than area I and II, backscattering in the arsenic EXAFS was assumed to be from iron atoms. Because these two interatomic As-Fe distances associated with the valence state of arsenic species, mixtures of $\mathrm{As}^{\mathrm{III}}$ and $\mathrm{As}^{\mathrm{V}}$ where overlapping backscattering shells caused these complicated geometries and hard to identify the each of the iron backscattering shells among all of the samples. However, there is a relative evident third shell with distances around $2.9 \AA$ at higher doses reaction after 60 days, indicating a substantial bridging formed at this time scale.

2. Root et al, Arsenic sequestration by sorption processes in high-iron sediments. Geochimica et Cosmochimica Acta 2007, 71, 5782-5803. 


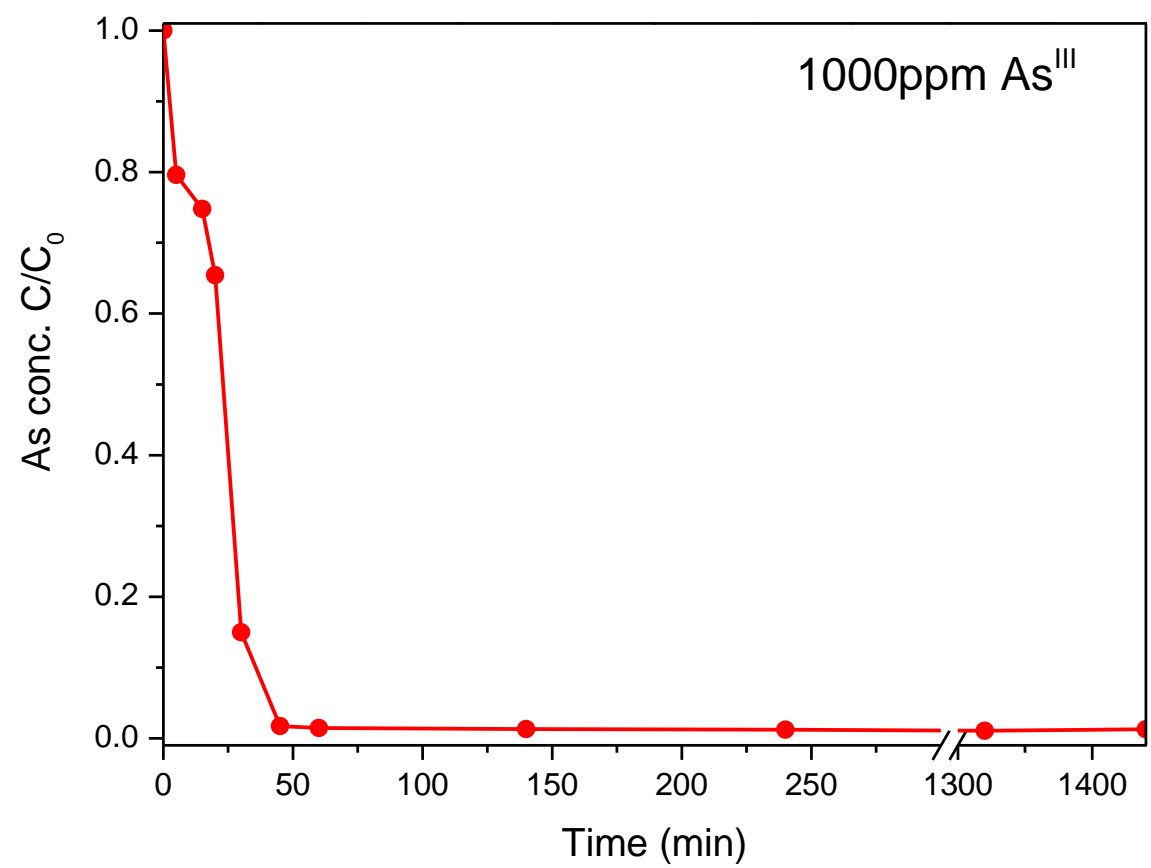

Figure S6. The reaction curve for high arsenic concentration sequestration with same ratio of $0.5 \mathrm{~g} / \mathrm{L} \mathrm{nZVI}$ 


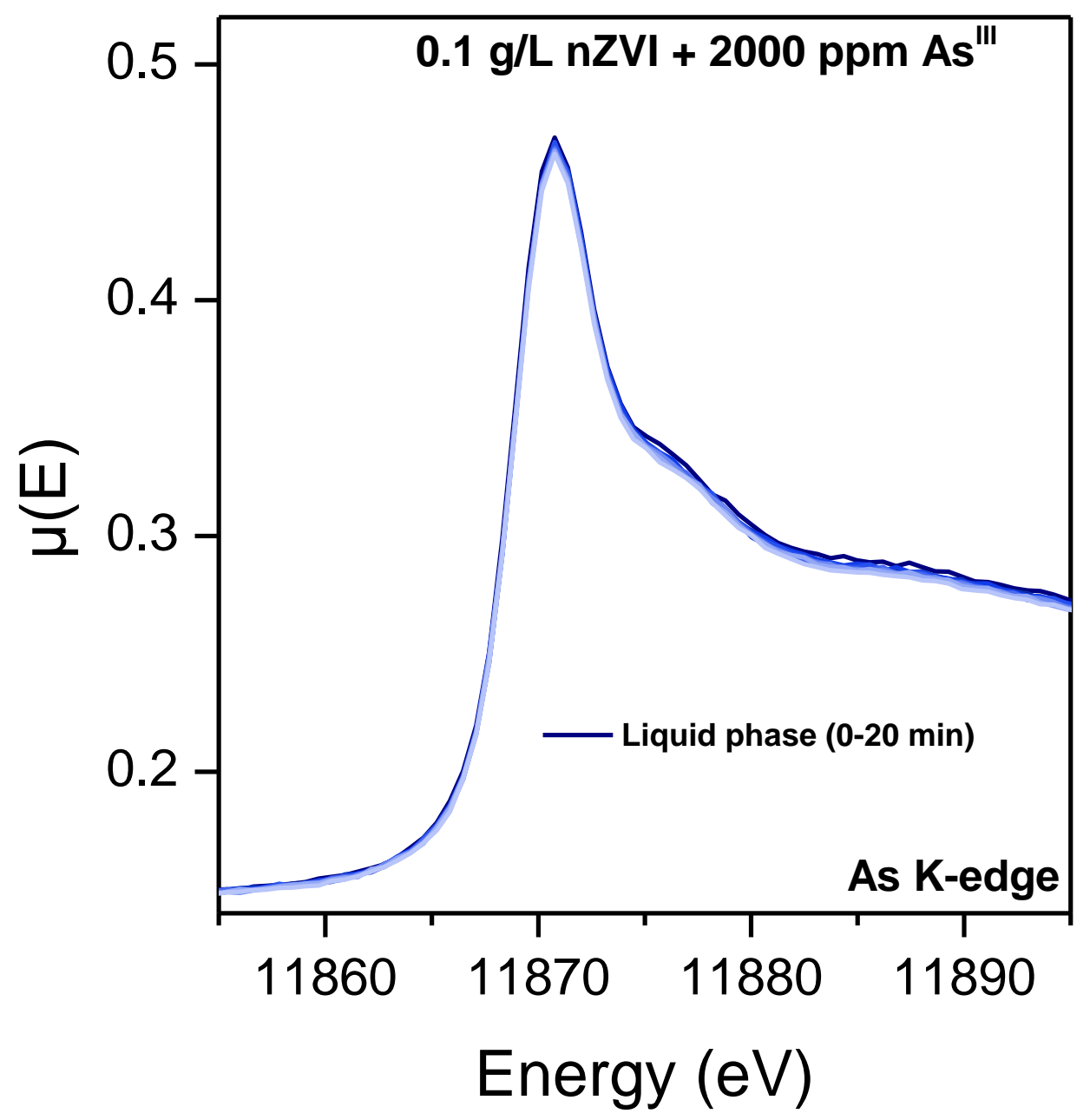

Figure S7. QEAXFS experiment at $0.1 \mathrm{~g} / \mathrm{L} \mathrm{nZVI} \mathrm{loading}$ 